\title{
Recubrimientos porosos de dióxido de titanio sintetizados por oxidación anódica
}

\section{Porous titanium dioxide coatings synthesized by anodic oxidation}

\author{
Hernán Darío Traid ${ }^{1,2}$, Anabela Natalia Dwojak ${ }^{1,2}$, María Laura Vera ${ }^{1,2}$, \\ Alicia Esther Ares ${ }^{1,2}$, Marta Irene Litter ${ }^{3,4}$
}

\footnotetext{
${ }^{1}$ Instituto de Materiales de Misiones - IMAM - CONICET/UNaM - CP: 3300, Posadas, Misiones, Argentina.

${ }^{2}$ Facultad de Ciencias Exactas, Químicas y Naturales - UNaM - CP: 3300, Posadas, Misiones, Argentina.

${ }^{3}$ Gerencia Química - CAC/CNEA - CP: 1650, San Martín, Buenos Aires, Argentina.

${ }^{4}$ Instituto de Investigación e Ingeniería Ambiental - 3iA - UNSAM - CP: 1650, San Martín, Buenos Aires, Argentina. e-mail: traidhernan@gmail.com; anabelanataliadwojak@gmail.com; veramalau@gmail.com; aares@fceqyn.unam.edu.ar; marta.litter@gmail.com
}

\section{RESUMEN}

$\mathrm{El} \mathrm{TiO}_{2}$ es uno de los materiales más utilizados en el proceso de fotocatálisis heterogénea debido a su disponibilidad comercial, bajo costo, baja toxicidad y elevada actividad fotocatalítica. Uno de los métodos más eficientes y económicos para obtener recubrimientos de $\mathrm{TiO}_{2}$ es la oxidación electrolítica por plasma. Durante este proceso de anodización, se hace circular corriente continua entre el ánodo y el cátodo inmersos en una solución de electrolito, a voltajes elevados que inducen la formación de arcos eléctricos (spark discharge) en la superficie del ánodo. En el presente trabajo se sintetizaron recubrimientos porosos de $\mathrm{TiO}_{2}$ por oxidación electrolítica por plasma, empleando ácido sulfúrico $1 \mathrm{M}$ como electrolito, cátodo de Pt, titanio Grado 2 como ánodo y una densidad de corriente máxima de $3250 \mathrm{~A} \mathrm{~m}^{-2}$. Se estudió la influencia del voltaje (90-180 V) y el acabado superficial del ánodo (con y sin pulido metalográfico) sobre la morfología y estructura cristalina de los recubrimientos. Se registró la evolución temporal de la densidad de corriente y el voltaje. La morfología de los óxidos se observó por microscopía electrónica de barrido. Las fases cristalinas se determinaron por difracción de rayos X con incidencia rasante. Las curvas de la densidad de corriente y el voltaje en función del tiempo muestran un comportamiento característico del crecimiento galvanostático-potenciostático de óxidos anódicos, con fluctuaciones propias del fenómeno de spark discharge. Las micrografías de los óxidos mostraron poros con un diámetro promedio 60-160 nm, que aumentó con el voltaje. Los difractogramas indicaron la presencia de las fases anatasa y rutilo del $\mathrm{TiO}_{2}$, con disminución de la fracción anatasa con el incremento del voltaje. No se observó influencia del acabado superficial sobre la morfología y la cristalinidad de los recubrimientos. Los óxidos sintetizados presentan características favorables como material de potencial aplicación en procesos de fotocatálisis heterogénea.

Palabras clave: dióxido de titanio, tratamiento de aguas, ácido sulfúrico, fotocatálisis heterogénea, oxidación electrolítica por plasma.

\section{ABSTRACT}

Titanium dioxide is one of the most used materials in heterogeneous photocatalysis due to its commercial availability, low cost, chemical stability, low toxicity and high photocatalytic activity. One of the most efficient and economical methods to obtain $\mathrm{TiO}_{2}$ coatings is the plasma electrolytic oxidation. In this anodization process, a direct current is circulated between a cathode and an anode immersed in an electrolyte solution, at a high voltage that induces the formation of spark discharges on the surface of the anode.

In the present work, porous $\mathrm{TiO}_{2}$ coatings were obtained by plasma electrolytic oxidation, using $1 \mathrm{M}$ sulfuric acid as the electrolyte, a Pt cathode, titanium Grade 2 as the anode and a maximum current density of $3250 \mathrm{~A}$ $\mathrm{m}^{-2}$. The influence of voltage (90-180 V) and surface finishing (with and without metallographic polishing) on the morphology and crystalline structure was studied. The temporal evolution of voltage and current density were recorded. The morphology of the oxides was observed by scanning electron microscopy, and the 
crystalline phases were determined by grazing incidence $\mathrm{X}$-ray diffraction.

The curves of current density and voltage showed behaviors characteristic of galvanostatic-potenciostatic grown of anodic oxides, with fluctuations as the result of the spark discharge condition. Micrographs of the oxides showed pores with an average diameter between 60 and $160 \mathrm{~nm}$, which increased at higher voltages. The diffractograms showed the presence of the anatase and rutile phases of $\mathrm{TiO}_{2}$, with a decrease in the anatase fraction at the higher voltages. No influence of surface finishing was observed on the morphology and crystallinity of the coatings.

The synthesized oxides show favorable characteristics as potential materials for heterogeneous photocatalytic processes.

Keywords: titanium dioxide, water treatment, sulfuric acid, heterogeneous photocatalysis, plasma electrolytic oxidation.

\section{INTRODUCCION}

El proceso de fotocatálisis heterogénea aplicado al tratamiento de aguas, tanto residuales como las destinadas al consumo humano, ha sido extensamente estudiado en las últimas décadas [1-3] De los semiconductores utilizados en este proceso, el dióxido de titanio $\left(\mathrm{TiO}_{2}\right)$ es el más empleado debido a sus características intrínsecas que lo hacen un excelente fotocatalizador: bajo costo, elevada estabilidad química, producción de huecos fuertemente oxidantes y elevada actividad fotocatalítica, particularmente de la fase cristalina anatasa [1]. Dada la naturaleza superficial del proceso de fotocatálisis heterogénea, es deseable que la superficie del $\mathrm{TiO}_{2}$ sea porosa, de modo de mejorar su actividad fotocatalítica incrementando el área superficial [1]. Por otro lado, se busca que en el material estén presentes las dos fases cristalinas del $\mathrm{TiO}_{2}$ más comunes, anatasa y rutilo, ya que, por un lado, la anatasa es reconocida como la de mayor actividad fotocatalítica y, por el otro, se ha demostrado que la presencia de 30 a $40 \%$ de rutilo puede aumentar la eficiencia fotocatalítica [1-4]

Las técnicas empleadas para la obtención del $\mathrm{TiO}_{2}$ son muy variadas: pulverización por plasma, sinterizado, procesos sol-gel, oxidación anódica con corriente o potencial constante, oxidación térmica, implantación de iones, etc. [1]. Un modo eficiente, económico y ambientalmente amigable de obtener recubrimientos porosos de $\mathrm{TiO}_{2}$ sobre titanio es el empleo de una variante de la técnica de oxidación anódica, conocida como oxidación electrolítica por plasma (en inglés: plasma electrolytic oxidation (PEO), microarc oxidation $(M A O)$ o anodic spark deposition $(A S D))$ [1,2]. Durante este proceso de anodización, se hace circular corriente continua entre el ánodo y el cátodo inmersos en una solución de electrolito a voltajes que superan un valor crítico $(>90 \mathrm{~V})$, lo cual induce la formación de arcos eléctricos (spark discharge) en la superficie del ánodo. Estos arcos eléctricos se inician donde el óxido preexistente es débil, y se produce una ruptura dieléctrica (breakdown) dando lugar a densidades de corriente y temperaturas locales elevadas (próximas a los $8000{ }^{\circ} \mathrm{C}$ ). Estas condiciones permiten obtener recubrimientos porosos de elevada área superficial e inducen la formación de óxidos cristalinos [1,2]. Los parámetros que más afectan las características (morfología, estructura cristalina, etc.) del óxido formado en el ánodo son la naturaleza, concentración, $\mathrm{pH}$ y temperatura del electrolito utilizado, el voltaje aplicado, el tiempo de anodización, y la densidad de corriente $[9,1-5]$

El objetivo del presente trabajo fue la obtención de recubrimientos de $\mathrm{TiO}_{2}$ porosos por medio de oxidación electrolítica por plasma evaluando el efecto del voltaje y el tratamiento superficial sobre la morfología y estructura cristalina de los óxidos, con la finalidad de su aplicación en procesos de fotocatálisis.

\section{MATERIALES Y MÉTODOS}

\subsection{Preparación del sustrato}

Se utilizaron como sustrato probetas de titanio grado 2 (según ASTM B367 [1]) de $3 \times 2 \times 0,2 \mathrm{~cm}$. Para evaluar la influencia de la terminación superficial del sustrato en las propiedades de los óxidos, algunas probetas se prepararon metalográficamente y otras se dejaron con el óxido natural (Figura 1). A las probetas que tuvieron preparación superficial, se las incluyó en acrílico autocurable (Subiton), se desbastaron manualmente con papeles abrasivos de SiC (Köln) de granulometría creciente, desde \#120 hasta \#1500, y luego se pulieron durante 30 minutos con pasta de diamante de $1 \mu \mathrm{m}$ (Praxis) lubricada con etilenglicol (Cicarelli) en una máquina pulidora a $250 \mathrm{rpm}$. Luego del pulido, se separaron las probetas del acrílico, se limpiaron con agua y detergente, luego con alcohol y, finalmente, se secaron con aire caliente.

Previamente a la oxidación, a todas las probetas se les colocó una barrera de acrílico en su parte superior con el objetivo de controlar el área de oxidación y proteger las conexiones de la celda electroquímica. 


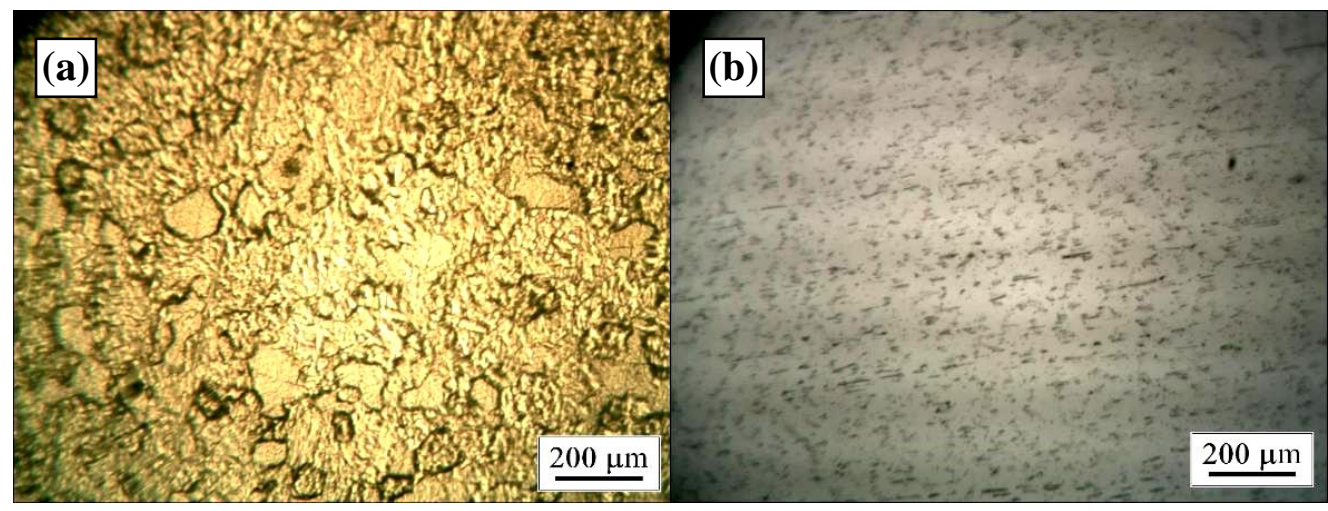

Figura 1: Micrografías ópticas: (a) probeta sin pulir, óxido natural, (b) probeta pulida con diamante $1 \mu \mathrm{m}$.

\subsection{Oxidación anódica}

Se utilizaron como cátodo dos láminas de platino distanciadas a $5 \mathrm{~cm}$ del ánodo de titanio, y ácido sulfúrico 1 M (Cicarelli) a una temperatura de $25^{\circ} \mathrm{C}$ como electrolito. La anodización se realizó con dos fuentes de corriente continua conectadas en serie (JMB LPS360DD y Kaise HY3005D-3), iniciando a una densidad de corriente constante de $3250 \mathrm{~A} \mathrm{~m}^{-2}$ (modo galvanostático) hasta el voltaje preestablecido, y continuando luego a voltaje constante (modo potenciostático) en condiciones de spark discharge. Los voltajes fueron 90, 120, 150 y $180 \mathrm{~V}$ para las probetas pulidas y 120 y $180 \mathrm{~V}$ para probetas sin pulir, a fin de evaluar el efecto de la preparación superficial del sustrato a dos voltajes diferentes. El tiempo de anodización máximo fue de 5 minutos. Finalizada la anodización, las probetas se enjuagaron con agua, alcohol y luego se secaron con aire caliente. En todos los casos, se registró la densidad de corriente y el voltaje en función del tiempo de anodización.

Las probetas se nombraron con una letra $\mathrm{V}$ y un número, indicando el voltaje empleado en la anodización, seguido de una sigla que indica la preparación superficial, "P" para las probetas pulidas y "NP" para las probetas no pulidas. Por ejemplo, la probeta V120-P corresponde a una probeta pulida oxidada a $120 \mathrm{~V}$.

\subsection{Caracterización}

La morfología de los recubrimientos se observó por microscopía electrónica de barrido (MEB), empleando un microscopio Carl Zeiss Supra 40. Las micrografías se analizaron empleando el programa ImageJ [1] para determinar el tamaño promedio de los poros. Las fases cristalinas presentes en los recubrimientos se determinaron por difracción de rayos X con un difractómetro Panalytical, Modelo Empyrean con detector PIXCEL3D. Como se trata de recubrimientos delgados $(\sim 500 \mathrm{~nm})$, se empleó incidencia rasante de $1^{\circ}$, para reducir la profundidad de penetración de los rayos $\mathrm{X}$ y tener mayor sensibilidad en los picos de las estructuras correspondientes a los recubrimientos [1]. La fracción anatasa fue determinada mediante la ecuación (1)

$$
X_{\mathrm{A}}=1 /\left[1+2,18\left(I_{\mathrm{R}} / I_{\mathrm{A}}\right)\right] \pm 2 \%
$$

donde $X_{\mathrm{A}}$ es la fracción molar de anatasa y $I_{\mathrm{A}}$ e $I_{\mathrm{R}}$ son las áreas de los picos más intensos correspondientes a las fases cristalinas anatasa (101) y rutilo (110), respectivamente [1].

\section{RESULTADOS Y DISCUSIÓN}

\subsection{Evolución del voltaje y de la densidad de corriente}

En la Figura 2 (a) se presenta la evolución temporal del voltaje y la densidad de corriente durante la anodización de la probeta V150-P. Se observa un rápido crecimiento del voltaje de la celda hasta que se alcanza el potencial establecido, momento a partir del cual se observa una caída en la densidad de corriente. Éste es el comportamiento típico de crecimientos galvanostáticos-potenciostáticos: la formación y el engrosamiento de la capa de óxido producen un aumento gradual en la resistencia al paso de la corriente, aumentando el voltaje $\mathrm{y}$, establecido el voltaje final, se genera un descenso progresivo en la densidad de corriente hasta alcanzar un valor de equilibrio entre las reacciones de disolución y crecimiento de la película anódica [1,2]; la medida de estos parámetros indica que al incrementar el voltaje empleado en la anodización mayor es la densidad de corriente de equilibrio registrada (Figura 2 (b)) [1]. En ambas Figuras, 2 (a) y (b), las fluctuaciones que se observan en las curvas de voltaje y de densidad de corriente serían resultado del ciclo sustrato desnudo- 
oxidación-ruptura propio de la oxidación electrolítica por plasma [25].

(a)

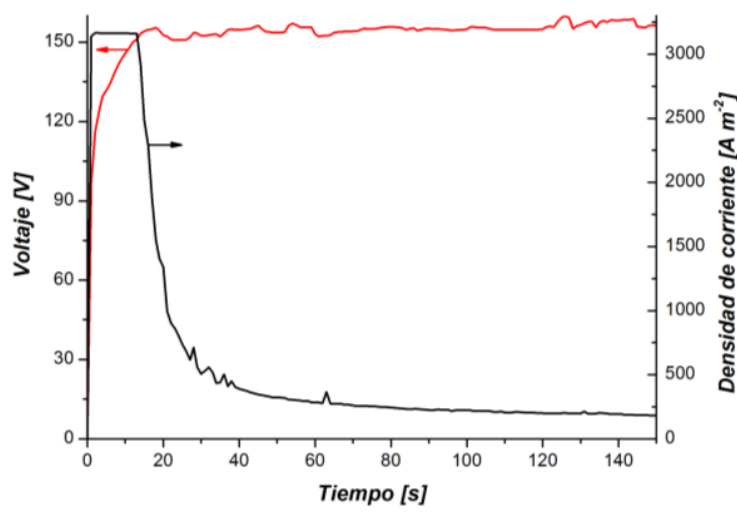

(b)

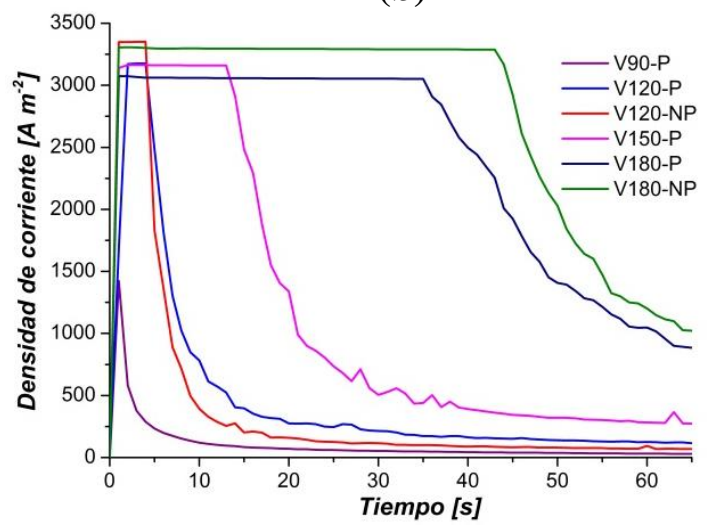

Figura 2: Evolución temporal de los parámetros de síntesis: (a) Voltaje (eje izquierdo) y densidad de corriente (eje derecho) para la probeta V150-P, (b) Densidad de corriente durante la síntesis de los recubrimientos.

En todos los casos se logró observar experimentalmente la presencia de arcos eléctricos en la superficie del ánodo (spark discharge) y la presencia, durante todo el proceso, de burbujas de hidrógeno y oxígeno en la superficie de cátodo y ánodo, respectivamente [11].

Por otra parte, si bien en la Figura 2 (b) se observan diferencias entre las probetas pulidas y sin pulir, no es clara la influencia del pulido sobre la corriente de equilibrio.

\subsection{Microscopía electrónica de barrido}

En la Figura 3 se presentan las micrografías de las probetas con los recubrimientos sintetizados, en las que se puede apreciar, en gris más oscuro, la presencia de poros distribuidos en la superficie. Puede observarse que los poros presentan los bordes elevados (tipo "cráter"), lo que sería evidencia de que, durante la formación del óxido, se produjo la evolución de oxígeno gaseoso [16,1]. Esto es consistente con el burbujeo sobre el ánodo observado experimentalmente.

En las Figuras 3 (a) a (f), puede observarse que al aumentar el voltaje aumenta el tamaño de los poros. Los valores calculados se presentan en la Tabla 1. Resultados similares de aumento del tamaño de poros con el voltaje fueron observados por Kuromoto y col. [16] para oxidaciones realizadas con ácido fosfórico.

En cuanto a la evolución de la morfología con el voltaje, se observa que el recubrimiento obtenido a $90 \mathrm{~V}$ (Figura 3 (a)) corresponde a un estado inicial de formación de poros producto del inicio del spark, dado que hay poros abiertos y cerrados rodeados de "valles" planos. Sin embargo, en el recubrimiento obtenido a $120 \mathrm{~V}$ (Figuras 3 (c) y (e)) la distribución de poros abiertos es uniforme en toda la superficie. Con el aumento del voltaje, en el recubrimiento obtenido a $150 \mathrm{~V}$ (Figura 3 (b)), se observan poros más grandes e interconectados, efecto que se acentúa a los $180 \mathrm{~V}$ (Figuras 3 (d) y (f)), producto del enérgico spark que hace colapsar a los poros más pequeños a expensas del crecimiento de los más grandes.

Por otro lado, se observa que el acabado superficial no tiene efectos apreciables sobre la morfología (distribución y tamaño de poros) de los recubrimientos (cf. en Figura 3, (c) vs. (e) y (d) vs. (f)).

Tabla 1: Diámetro promedio de los poros y fracción anatasa de los recubrimientos sintetizados.

\begin{tabular}{l|c|c}
\hline PROBETA & DIÁMETRO [NM] & FRACCIÓN ANATASA \\
\hline$V 90-P$ & 56 & 0,876 \\
\hline$V 120-P$ & 81 & 0,807 \\
\hline$V 120-N P$ & 86 & 0,865 \\
\hline$V 150-P$ & 105 & 0,377 \\
\hline$V 180-P$ & 160 & 0,149 \\
\hline$V 180-N P$ & 153 & 0,106 \\
\hline
\end{tabular}




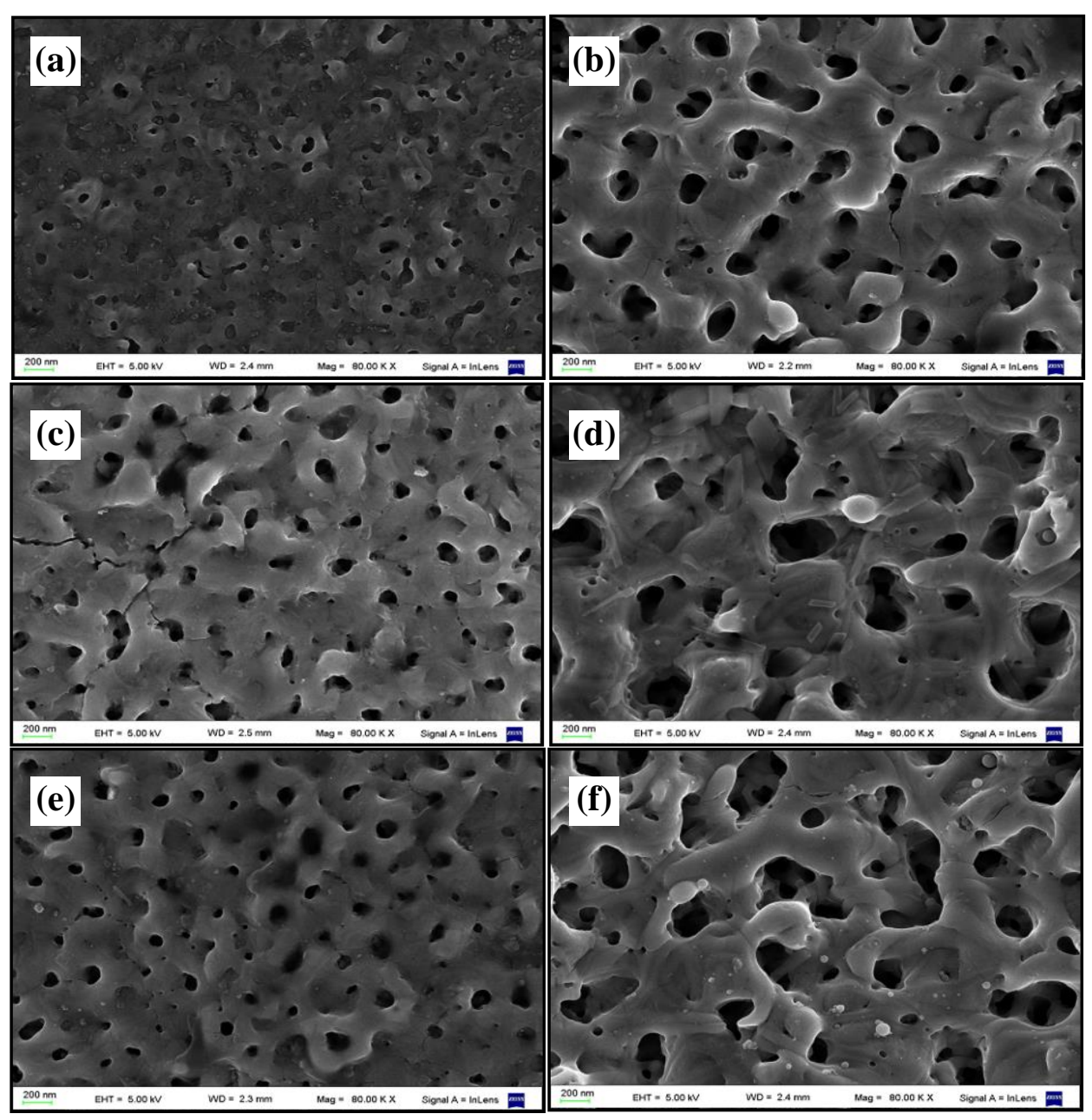

Figura 3: Micrografias electrónicas de barrido de las probetas: (a) V90-P, (b) V150-P, (c) V120-P, (d) V180-P, (e) V120-NP, (f) V180-NP

\subsection{Difracción de rayos $\mathrm{X}$}

En la Figura 4 se presentan los difractogramas de rayos X de incidencia rasante de los recubrimientos sintetizados y del sustrato pulido sin oxidar.

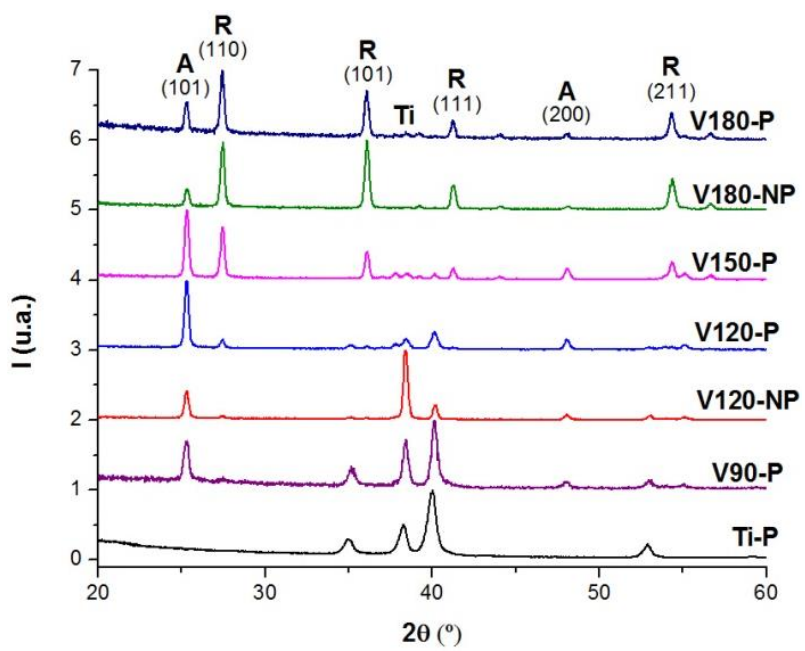

Figura 4: Difractogramas de los recubrimientos y el sustrato. A: anatasa; R: rutilo; Ti: titanio (sustrato).

En todos los difractogramas correspondientes a las probetas recubiertas, se puede observar la presen- 
cia de las fases anatasa y rutilo del $\mathrm{TiO}_{2}$. Esto implica que las condiciones de síntesis permitieron la obtención de recubrimientos cristalinos en todos los casos, evitando a priori la necesidad de tratamientos térmicos post-anodización que induzcan la cristalización de los óxidos.

Se aprecia que un incremento en el voltaje conlleva una disminución progresiva de la intensidad del pico del sustrato (Ti). Esto podría ser resultado del incremento en el espesor de $\mathrm{TiO}_{2}[9,16]$. Por otra parte, un incremento en el voltaje de anodización reduce la fracción anatasa de los recubrimientos (Tabla 1), observable en la Figura 4 por el incremento de la intensidad de los picos de rutilo y la disminución de la intensidad de los picos de anatasa. Esto se debería a que el aumento del voltaje promueve condiciones de spark más enérgicos que van transformando progresivamente la anatasa a la fase rutilo más estable.

Por otra parte, la preparación superficial no tiene un efecto claro sobre la fracción anatasa de los óxidos.

\section{CONCLUSIONES}

Se obtuvieron recubrimientos porosos y cristalinos de $\mathrm{TiO}_{2}$ por medio de oxidación electrolítica por plasma.

Se observó que el incremento del voltaje de anodizado aumenta el tamaño de los poros de los recubrimientos y reduce la fracción anatasa frente a la de rutilo, mientras que la preparación superficial del sustrato no mostró un efecto claro sobre la morfología ni la fracción anatasa de los óxidos. Deberá realizarse un mayor número de experimentos a fin de establecer claramente su efecto.

En vistas de la aplicación deseada, es decir, en procesos de fotocatálisis heterogénea para el tratamiento de aguas, los voltajes de oxidación que resultan más promisorios son 120 y $150 \mathrm{~V}$ debido al tamaño de poros y la fracción anatasa de los recubrimientos obtenidos. Futuros ensayos permitirán precisar el efecto de la preparación superficial sobre la actividad fotocatalítica de los recubrimientos.

\section{AGRADECIMIENTOS}

Los autores agradecen al Consejo Nacional Investigaciones Científicas y Técnicas (CONICET) y a la Agencia Nacional de Promoción Científica y Tecnológica (ANPCyT), de Argentina, por el financiamiento de la presente investigación, y al Dr. Daniel Vega del Laboratorio de Difracción de Rayos X, Departamento Física de la Materia Condensada, Gerencia de Investigación y Aplicaciones, del Centro Atómico Constituyentes de la Comisión Nacional de Energía Atómica de Argentina.

\section{BIBLIOGRAPHY}

[1] LITTER, M.I., "Heterogeneous photocatalysis: transition metal ions in photocatalytic systems", Applied Catalysis B: Environmental, v. 23, n. 2, pp. 89-114, 1999.

[2] LITTER M., QUICI N., "Photochemical Advanced Oxidation Processes for Water and Wastewater Treatment", Recent Patents on Engineering, v. 4, n. 3, pp. 217-241, 2010.

[3] FUJISHIMA, A., RAO, T.N., TRYK, D.A., "Titanium dioxide photocatalysis", Journal of Photochemistry and Photobiology C: Photochemistry Reviews, v. 1, n. 1, pp. 1-21, 2000.

[4] FUJISHIMA, A., ZHANG, X., "Titanium dioxide photocatalysis: present situation and future approaches”, Comptes Rendus Chimie, v. 9 n. 5, pp. 750-760, 2006.

[5] SU, C., HONG, B.Y., TSENG, C.M., "Sol-gel preparation and photocatalysis of titanium dioxide", Catalysis Today, v. 96, n. 3, pp. 119-126, 2004.

[6] HURUM, D.C., AGRIOS, A.G., GRAY, K.A., RAJH, T., THURNAUER, M.C., "Explaining the enhanced photocatalytic activity of Degussa P25 mixed-phase $\mathrm{TiO}_{2}$ using EPR", The Journal of Physical Chemistry B, v. 107, n. 19, pp. 4545-4549, 2003.

[7] SCANLON, D.O., DUNNILL, C.W., BUCKERIDGE, J., et al., "Band alignment of rutile and anatase $\mathrm{TiO}_{2}$ ", Nature materials, v. 12, n. 9, pp. 798-801, 2013.

[8] LUTTRELL, T., HALPEGAMAGE, S., TAO, J., KRAMER, A., et al., "Why is anatase a better photocatalyst than rutile? Model studies on epitaxial $\mathrm{TiO}_{2}$ films", Scientific reports, v. 4, 2014.

[9] TRAID, H.D., VERA, M.L., ARES, A.E., et al., "Advances on the synthesis of porous $\mathrm{TiO}_{2}$ coatings by anodic spark oxidation. Photocatalytic reduction of Cr (VI)”, Materials Chemistry and Physics, v. 191, pp. 106-113, 2017. 
[10] MIZUKOSHI, Y., MASAHASHI, N., "Photocatalytic activities and crystal structures of titanium dioxide by anodization: their dependence upon current density", Materials transactions, v. 51, n. 8, pp. 1443$1448,2010$.

[11] YEROKHIN, A.L., NIE, X., LEYLAND, A., MATTHEWS, A., DOWEY, S.J. "Plasma electrolysis for surface engineering”, Surface and Coatings Technology, v. 122, n. 2, pp. 73-93, 1999.

[12] STOJADINOVIĆ, S., VASILIĆ, R., PETKOVIĆ, M., et al., "Plasma electrolytic oxidation of titanium in heteropolytungstate acids", Surface and Coatings Technology, v. 206, n. 2, pp. 575-581, 2011.

[13] DIAMANTI, M.V., PEDEFERRI, M.P, "Effect of anodic oxidation parameters on the titanium oxides formation”, Corrosion Science, v. 49, n. 2, pp. 939-948, 2007.

[14] SONG, H.J., PARK, S.H., JEONG, S.H., et al., "Surface characteristics and bioactivity of oxide films formed by anodic spark oxidation on titanium in different electrolytes", Journal of Materials Processing Technology, v. 209, n. 2, pp. 864-870, 2009.

[15] TRAID, H.D., VERA, M.L., ARES, A.E. LITTER, M.I. "Porous titanium dioxide coatings obtained by anodic oxidation for photocatalytic applications", Procedia Materials Science, v. 9, pp. 619-626, 2015.

[16] KUROMOTO, N.K., SIMÃO, R.A., SOARES, G.A., “Titanium oxide films produced on commercially pure titanium by anodic oxidation with different voltajes", Materials Characterization, v. 58, n. 2, pp. 114$121,2007$.

[17] TRAID, H.D., VERA, M.L., ARES, A.E. LITTER, M.I., "Obtención de recubrimientos porosos de $\mathrm{TiO}_{2}$ por oxidación anódica para aplicaciones fotocatalíticas”, Avances en Ciencias e Ingeniería, v. 7, n. 1, pp. 19$25,2016$.

[18] DIKICI, T., EROL, M., TOPARLI, M., CELIK, E., "Characterization and photocatalytic properties of nanoporous titanium dioxide layer fabricated on pure titanium substrates by the anodic oxidation process", Ceramics International, v. 40, n. 1, pp. 1587-1591, 2014.

[19] MEYER, S., GORGES, R., KREISEL, G., "Preparation and characterisation of titanium dioxide films for catalytic applications generated by anodic spark deposition", Thin Solid Films, v. 450, n. 2, pp. 276-281, 2004.

[20] ASTM B367-04 Standard Specification for Titanium and Titanium Alloy Castings.

[21] RASBAND, W. S. 1997-2014. ImageJ, US National Institute of Health. Bethesda, Maryland, USA (http://imagej.nih.gov/ij/).

[22] LAMAS, D.G., "Incidencia rasante y reflectometría de rayos X”. Seminario en el marco de la I Escuela de la Asociación Argentina de Cristalografía. Posadas, Argentina. 10-11 Agosto, 2009.

[23] CRIADO, J., REAL, C., "Mechanism of the inhibiting effect of phosphate on the anatase $\rightarrow$ rutile transformation induced by thermal and mechanical treatment of $\mathrm{TiO}_{2}$ ", Journal of the Chemical Society, Faraday Transactions 1: Physical Chemistry in Condensed Phases, v. 79, n. 12, pp. 2765-2771, 1983.

[24] ZWILLING, V., DARQUE-CERETTI, E., BOUTRY-FORVEILLE, A., DAVID, D., PERRIN, M.Y., AUCOUTURIER, M., "Structure and physicochemistry of anodic oxide films on titanium and TA6V alloy", Surface and Interface Analysis, v. 27, n. 7, pp. 629-637, 1999.

[25] AMMAR, I.A., KAMAL, I., "Kinetics of anodic oxide-film growth on titanium-I. Acid media", Electrochimica Acta, v. 16, n. 9, pp. 1539-1553, 1971.

[26] VERA, M.L, "Obtención y caracterización de películas hemocompatibles de $\mathrm{TiO}_{2}$ ”, Tesis de Doctoral en Ciencia y Tecnología, Instituto Sábato. CNEA-UNSAM, Buenos Aires, Argentina, 2013.

[27] TEH, T.H., BERKANI, A., MATO, S., SKELDON, P., THOMPSON, G.E., HABAZAKI, H., SHIMIZU, K., "Initial stages of plasma electrolytic oxidation of titanium", Corrosion Science, v. 45, n. 12, pp. 2757-2768, 2003. 